\title{
Target Tracking Radar
}

\author{
Mahmoud Yahia Hussein ${ }^{1}$, Abdelrasoul Jabar Alzubaidi ${ }^{2}$ \\ 1, Department Of Electronics Engineering, 1SudanAcademy Of Sciences \\ 2, Electronics Engineering School, Sudan University Of science And technology
}

\begin{abstract}
Tracking radar systems are used to measure the target's relative position in range, azimuth angle, elevation angle, and velocity. Then, by using and keeping track of these measured parameters the radar can predict their future values. This paper proposes the measure a single target angle using sequential lobing technique. The information is used, in conjunction with Doppler, to centre the antenna beam to follow the target and maintain maximum power on the target. This is achieved in an angle tracking loop which measures the angle between the antenna bore sight and the line of sight to the target and attempts to correct for any differences. Azimuth and elevation angles are determined by measuring the difference between echo signals from two different positions.
\end{abstract}

Keywords: - Target tracking radar, antenna, sequential lobing ,angle tracking, pencil beam Azimuth angle, elevation angle

\section{INTRODUCTIONS}

A tracking radar measures the coordinates of a target and provides data which may be used to determine the target trajectory and to predict its future position. Target Tracking Radar TTR is important to military radars as well as to most civilian radars. In military radars, tracking is responsible for fire control and missile guidance, missile guidance is almost impossible without proper target tracking. Commercial radar systems, such as civilian airport traffic control radars, may utilize tracking as a means of controlling incoming and departing airplanes. Tracking techniques can be divided into range/velocity tracking and angle tracking. It is also customary to distinguish between continuous single-target tracking radars and multi-target track-while-scan (TWS) radars. Tracking radars utilize pencil beam (very narrow) antenna patterns. It is for this reason that separate search radar is needed to facilitate target acquisition by the tracker. In sequential lobing technique the radar beam is switched between different positions in order to gain improved angular information about the position of the target. Consider two beam positions witched between the left and right of a target. If the target is in the centre of the two beams then a signal of equal strength will be observed in each beam. If the target, on average, is growing in the left hand beam then the two beam positions are moved to the left until the signals are of equal strength in each of the beams. In this way the target is tracked.

\section{METHODOLOGY}

A. Hardware components:

The TTR is divided into eight major systems, each of which accomplishes one or more of the major functions that contribute to the overall operation of the radar. The functional block diagram Fig1depicts the eight major systems of the TTR. 


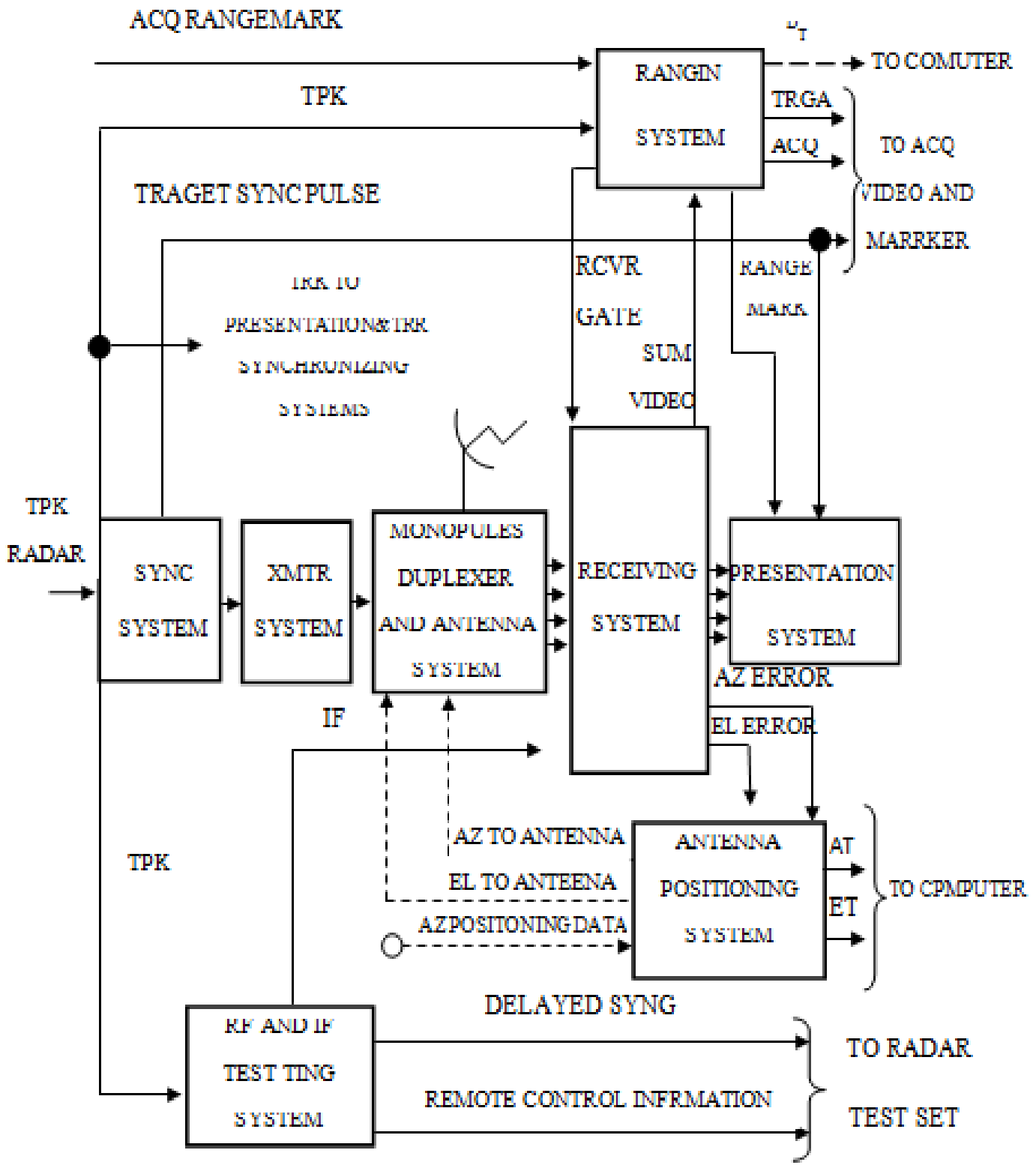

Figure 1: Functional block diagram of target track radar

\section{a. Synchronizing system}

The synchronizing system provides pulses that synchronize units of the TTR system. The "acquiring" of a target is a combined function of the acquisition radar system and the TTR and target ranging radar (TRR) systems: therefore, triggering the synchronizing system with the acquisition preknock pulse from the acquisition synchronizing system synchronizes the transmitter, range, and presentation systems of the TTR and TRR with the acquisition radar system. The synchronizing system Figure 1provides a target preknock pulse (TPK), target sync to the antenna positioning system. The sum IF signal is detected and applied to the track ranging system as the target range video signal. In addition, sum, azimuth errors. And elevation error video signals are applied to the presentation system for the CRT displays. Only those pulses received from the target being tracked are detected by the track receiver gate which is applied to the receiving system. This gating is necessary in order to reduce or eliminate echoes received from undesired targets in the vicinity.

\section{b. Transmitter system.}

The transmitter system Figure 1, triggered by transmitter sync, produces high power RF pulses at a frequency variable within the X-band. The RF pulses are coupled through the target track monopulse duplexer to the track antenna reflector assembly which beams the RF pulses into space. The long or short pulse mode of operation of the synchronizing system determines duration of the transmitted RF pulses. 


\section{c. RF (monopulse duplexer) and antenna system.}

The purpose of this system is to radiate the high power RF energy, generated in the transmitting system, into space and to receive the energy that is reflected when a target intercepts the RF beam. In most tracking radars, lobe switching or conical scanning is used to obtain target position information, and several pulses must be used to determine target azimuth and elevation .However, in the monopulse system, which is employed in the TTR, target position information is obtained each time a pulse is transmitted, reflected, and received. The monopulse system employs two pairs of radiating feed horns that compare the amplitudes of a single returned pulse as it appears in the four feed horns. By subtracting the signal strength received by one pair of horns from the signal strength received by the adjacent pair, a difference-signal is obtained. The azimuth and elevation difference-signals are utilized by other systems of the radar to keep the antenna pointed at the target.

\section{d. Receiving system.}

The receiving system Figure 1 converts the X-band signals (sum, azimuth difference, and elevation difference RF signals) applied from the RF (monopulse duplexer) and antenna system, into60-megahertz IF signals. By using these three IF signals, the receiving system generates direct current azimuth and elevation error signals that are subsequently applied to the antenna positioning system. The sum IF signal is detected and applied to the track ranging system as the target range video signal. In addition, sum, azimuth errors. and elevation error video signals are applied to the presentation system for the CRT displays. Only those pulses received from the target being tracked are detected by the track receiver gate which is applied to the receiving system. This gating is necessary in order to reduce or eliminate echoes received from undesired targets in the vicinity.

\section{e. Ranging system.}

\section{(1) The ranging system}

Figure1determines the time between the transmitted pulse and the received target echo. Since the range of the target is proportional to the time interval between the transmitted pulse and the target echo, measuring the time interval determines the range. Three means are provided for controlling the measuring circuits after the target is acquired: manual, aided. and automatic. Manual and aided ranging can be tested for acquiring a target. All three ranging methods can be used after the target is acquired by TTR to provide range data for the computer. The circuits of the ranging system are triggered by the preknock pulse, during normal operation. During calibration, a calibration sync pulse is applied to the target ranging system to replace the preknock pulse. During normal operation, the preknock pulse initiates operation of the ranging system. After a controllable time delay, the ranging system produces the receiver gate (RCVR gate) which is sent to the receiver system and can be centered on the sum target video to give an indication of target slant range (DT).

\section{(2) During the designation and acquiring of a target}

The acquisition radar range and azimuth data are transferred to TTR. The TTR uses this data (acq range mark and az position data) to slew the TTR range and antenna system to the range and azimuth of the designated target. When the elevation operator manually "acquires" the target is in the TTR beam and all three target coordinates (AT, ET, and DT) now appear on the TTR indicators. These coordinates (AT, ET, and DT) can now be sent to the computer. Also, when the track antenna and range system slews to the designated target position, the track range gate (TRGA) and acq track range mark (acq TRMK) are sent to the video and marker circuits in the battery control van. This causes the electronic cross to move to the coordinate of the designated target. The TRGA produces the radial line and the acq TRMK produces the arc of the electronic cross. Back in the radar control trailer, presentation of the target coordinates enables the three track operators to manually track the target. Once the target is manually tracked in AT, ET, and DT, automatic tracking may begin.

\section{f. Antenna positioning system.}

The function of this system is to correct the antenna position so that it points directly at the target at all times. Manual and aided positioning are employed for acquiring the target. Automatic positioning keeps the antenna on-target at all times without the aid of the operators. The antenna is positioned by direct current azimuth and elevation error signals Figure1 provided by the receiving system.

\section{g. Presentation system.}

The target track radar presentation system consists of three A scan indicators which provide visual tracking information in three coordinates: azimuth, elevation, and range. These indicators are used in manual and manual-aided tracking of a target and supply helpful information during automatic tracking. Thus, the presentation system is the visual link between the target and the TTR operators. Three 5-inch A type scopes and 
a B scope appear on the target tracking control console in the trailer mounted tracking station. The B scope, which provides a visual link between the acquisition and track system,

\section{h. IF and RF testing systems.}

\section{(1) IF test system.}

The IF test system Figurelproduces a 60-megahertz IF test signal for testing the TTR system. The target preknock pulse from the synchronizing system triggers the IF tests system. A 60-megahertz IF test signal, capable of variable delay, is applied to the main IF amplifiers in the receiver system when tests, calibrations, and adjustments are being performed.

\section{(2) RF test system.}

The RF test system Figure 1remotely controls the operation of the radar test set group during testing of the overall RF performance of $\mathrm{t}$ he TTR system. The target preknock pulse is applied to the RF test system to develop a delayed sync pulse which triggers the radar test set group into operation.

The radar test set group generates test signals used to check the receiver system. Controls in the RF test system vary the frequency, amplitude, and apparent range of these test signals by applying remote control information to the radar test set group.

\section{B. Software (Single Target Tracking):}

\section{Angle tracking:}

There are different ways to measure the target angle; we used sequential lobing technique in our study to measure it.

\section{Sequential Lobing}

Sequential lobing has a tracking accuracy that is limited by the pencil beam width used and by the noise caused by either mechanical or electronic switching mechanisms. The pencil beam used in sequential lobing must be symmetrical (equal azimuth and elevation beam widths).

Tracking is achieved by continuously switching the pencil beam between two pre-determined symmetrical positions around the antenna's Line of Sight (LOS) axis. The LOS is called the radar tracking axis, as illustrated in Figure 2.

\section{CONCEPT OF DETERMINING THE ANGLE BY SEQUENTIAL LOBING}

1. When the target is tracked on the tracking axis, as the case in Figure $\mathbf{2} \mathbf{a}$, the voltage difference is zero and the error signal equal zero too.

2. When the target is off the tracking axis, as in Figure $\mathbf{2} \mathbf{~ b}$, a nonzero error signal is produced. The sign of the voltage difference determines the direction in which the antenna must be moved.

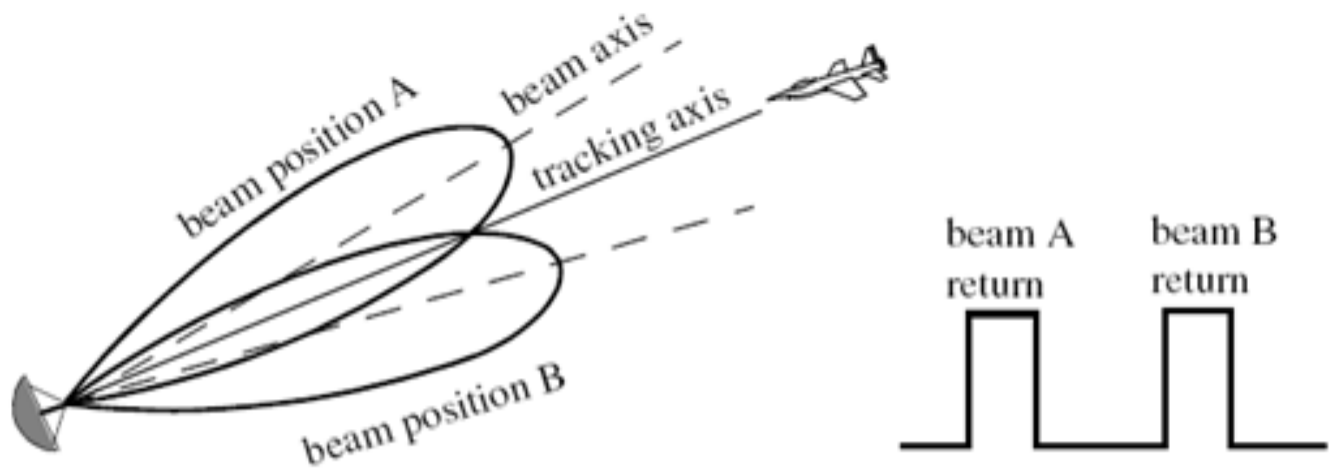

(a) Target is located on track axis. 

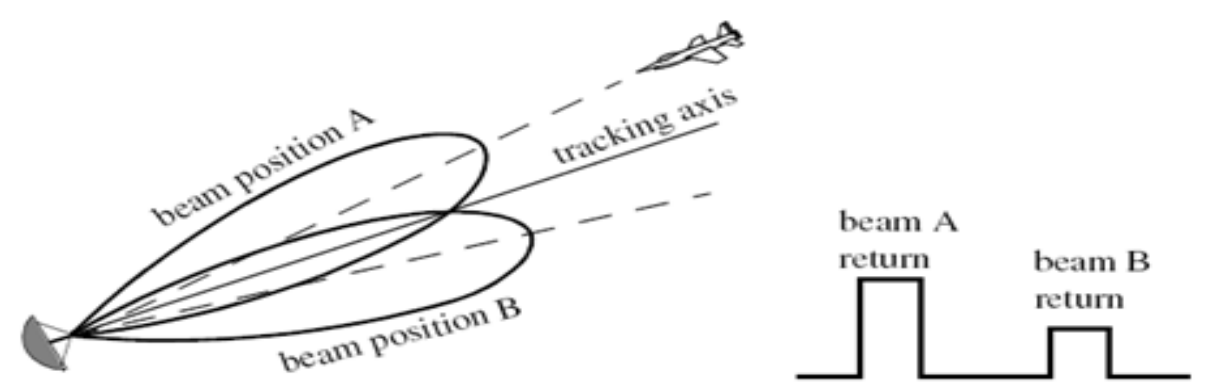

(b) Target is off track axis.

Figure 2: Sequential lobing technique.

\section{Flowchart}

RESULTS

1. $\quad \mathrm{V} 1=$ voltage from upper beam (lobe)

2. $\quad \mathrm{V} 2=$ voltage from lower beam (lobe)

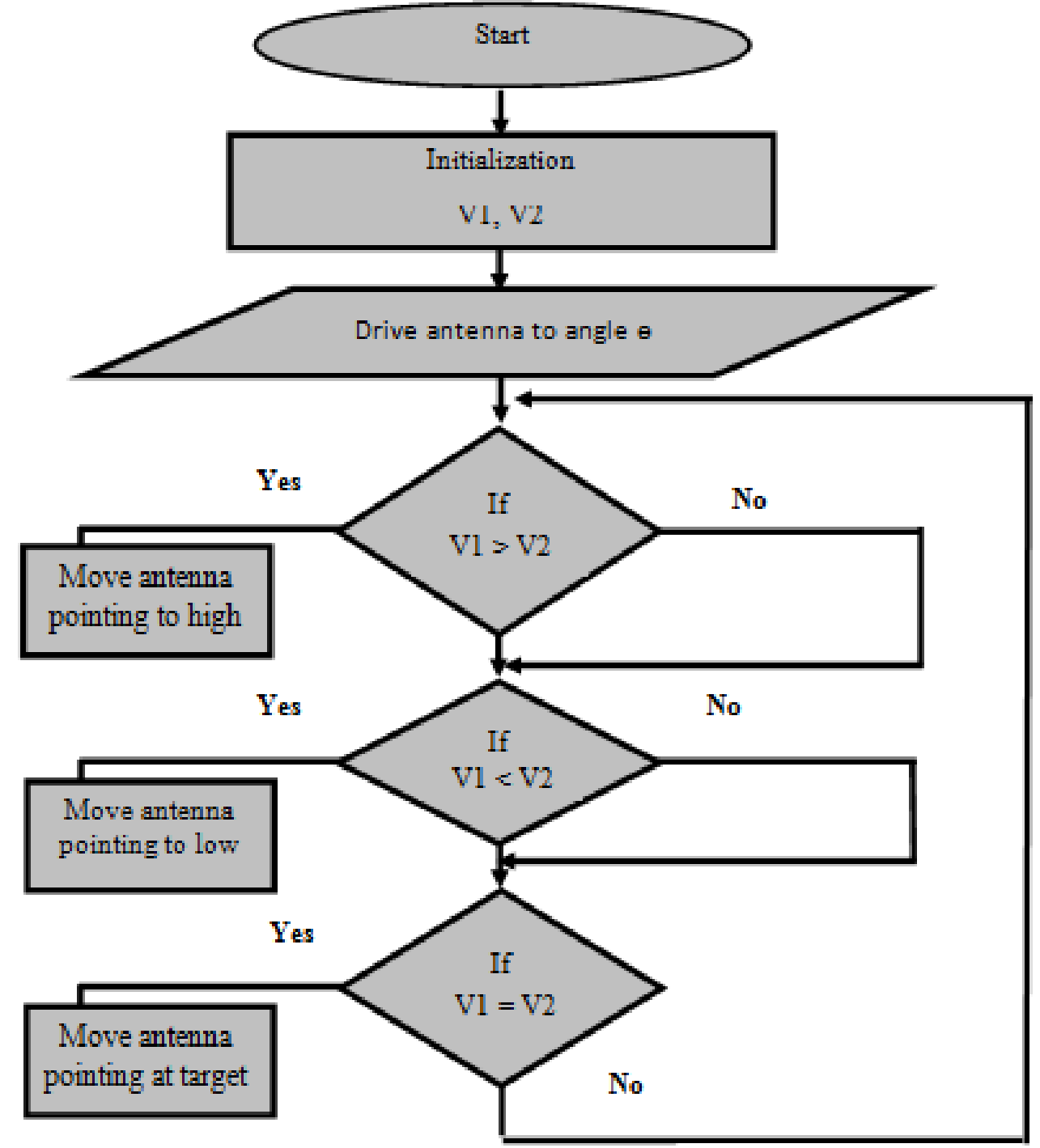

Figure 3: Flowchart to sequential lobing technique 


\section{CONCLUSION}

In this project a theoretical analysis of the sequential lobing technique for target angle tracking is presented. The signal power received by each antenna beam is assumed to pass through a logarithm. The difference of the voltage amplitudes between the two positions gives the angular measurement error and the beam is moved to the direction in which the amplitude of the voltage is larger .If the amplitude of the voltages corresponding to the two positions of the target are the same, the target is said to be on the switching axis.

\section{REFERENCES}

[1] Merrill I. Skolnik, Introduction to Radar Systems, 3rd Edition, pp. 210-238, Mc Graw-Hill, New York, NY, 2001.

[2] David K. Barton, Radar System Analysis, pp.83-89, 327-331, Artech House, Dedham, MA, 1979.

[3] Warren L. Stutzman and Gary A. Thiele, Antenna Theory and Design, 2nd Edition, pp. 80, 88-136, Wiley, Indianapolis, IN, 1998.

[4] David C. Jenn, "Tracking Arrays", Naval Postgraduate School, California, 2006.

[5] Bassem R. Mahafza CHAPMAN \& HALL/CRC Radar Systems Analysis and Design Using MATL

[6] http://www.radartutorial.eu/10.processing/sp21.en.html

[7] http://www.fas.org/man/dod-101/navy/docs/fun/part06.htm 